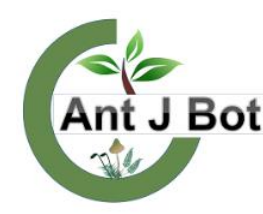

Received : 17.12.2018

Accepted : 02.01.2019

Online : :04.01.2019

\title{
Edible macrofungi determined in Gürpınar (Van) district
}

\author{
Ezelhan ŞELEM $^{1 *} \odot$, Ali KELEŞ ${ }^{1}{ }^{\odot}$, İsmail $\operatorname{ACAR}^{2} \odot$, Kenan DEMİREL ${ }^{3} \odot$ \\ ${ }^{1}$ Yüzüncü Yll University, Education Faculty, Department of Science and Mathematics Education, Van, Turkey

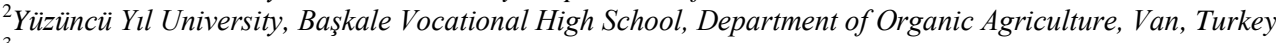 \\ ${ }^{3}$ Ordu University, Education Faculty, Department of Science and Mathematics Education, Van, Turkey \\ *ezelhanselem@hotmail.com
}

\section{Gürpınar (Van) yöresinde belirlenen yenilebilir makromantarlar}

\begin{abstract}
This study was carried out on wild edible macrofungi samples, collected within the boundaries of Gürpınar (Van) district between 2016 and 2017. As a result of necessary investigations related to macro and micromorphologies of the samples, 36 wild edible macrofungi species belonging to 11 families and 4 orders were determined. Eight of them belong to the phylum Ascomycota and 28 to Basidiomycota. Among the determined taxa Agaricus bisporus, A. campestris, A. urinascens, Pleurotus eryngii, $P$. ostreatus and P. populinus are collected and consumed by locals. Pleurotus eryngii is the only economically important macrofungi in the region.
\end{abstract}

Key words: Biodiversity, edible mushrooms, Eastern Anatolia, Turkey

Özet: Bu çalışma 2016 ve 2017 yılları arasında Gürpınar (Van) ilçe sınırları içinde kalan bölgeden toplanan yabani yenilebilir makromantar üzerinde gerçekleştirilmiştir. Örneklerin makro ve mikromorfolojilerine ilişkin gerekli incelemeler sonucunda, 4 ordo ve 11 familyaya ait 36 yenilebilir yabani makromantar türü belirlenmiştir. Bunlardan 8 tanesi Ascomycota bölümüne, 28 tanesi ise Basidiomycota bölümüne aittir. Belirlenen taksonlardan Agaricus bisporus, A. campestris, A. urinascens, Pleurotus eryngii, $P$. ostreatus ve $P$. populinus yerel halk tarafindan toplanıp tüketilmektedir. Yörede ekonomik öneme haiz tek tür ise Pleurotus eryngii'dir.

Anahtar Kelimeler: Biyoçeşitlilik, yenilebilir mantarlar, Doğu Anadolu, Türkiye

\section{Introduction}

Turkey is a very rich country in terms of naturally growing macrofungal species, due to its unique flora and climatic conditions (Dündar et al., 2016), and currently comprises more than 2500 macrofungi (Kaya and Uzun, 2018). Fungi are an important group of organism for nature, and have a crucial role in recycling the organic matter. Beside the traditional consumption of naturally growing mushrooms as an important part of human diet, they are also used as a source of bioactive metabolites (Acharya et al., 2018). They are also emphasized by many researchers as valuable food source due to their fatty acid, vitamin, fiber, carbohydrate, protein and mineral contents (Demirbaş, 2001; Owaid et al., 2007; Türkekul, 2017). Because of their medical benefits, mushrooms have been consumed for centuries in many far eastern countries such as China and Japan (Manzi et al., 2001) as well as in many European and American countries (Boa, 2004). The findings of recent studies also indicate that many macrofungi accumulate heavy metals, radioactivity and some other toxic materials in their fruit bodies (Mat, 2000; Isıldak et al., 2004; Soylak et al.,2005; Kalać, 2010; Uzun et al., 2011; Kaya and Bağ, 2013; Korkmaz et al., 2016; Karapınar et al., 2017; Türkmen and Budur, 2018).

Gürpınar, where the research was conducted, is a district of Van province in Eastern Anatolian Region of Turkey. The district is the largest one in Turkey with a surface area of $4.063 \mathrm{~km}^{2}$ (Anonymus, 2018), and surrounded by Central district of Van and Özalp to the north, Gevaş and Çatak to the west, Hakkari to the south and Başkale (Van) to the east (Fig. 1). The research area lies within the IranoTuranean phytogeographical flora sector. Steppe vegetation is the dominant one in the region with distinct
Astragalus L. populations. Quercus L., Pinus L. and some Juniperus L. species form rare and mixed populations at higher elevations (Atalay, 1989). Along stream banks, Eleagnus Hill., Populus L., Malus Mill., Prunus L., Pyrus L. and Salix L. trees form small populations.

The climate of the research area is Mediterranean with an annual rainfall of $281 \mathrm{~mm}$ and an annual average temperature of $8.1^{\circ} \mathrm{C}$ (Bani and Adigüzel, 2008).

The study aims to determine the edible macrofungal composition and local consumption of them in the region, and to make a contribution to the mycobiota of Turkey.

\section{Materials and Method}

The research material was collected from suitable localities (Table 1) within the boundaries of Gürpınar (Van) district between the years 2016 and 2017. During field studies, macrophotographs of the macrofungi samples were taken at their natural habitats, and the diagnostic, descriptive and ecological characteristics were recorded. Local edibilty status of the collected samples were also investigated by asking the local people. Then the samples were transferred to the laboratory. Macroscopic and microscopic measurements and chemical investigations were performed in the laboratory. The specimens were identified by comparing the obtained data with the relevant literature (Moser, 1983; Breitenbach and Kränzlin, 1984-2000; Cappelli, 1984; Buczacki, 1989; Jordan, 1995; Bessette et al., 1997; Kränzlin, 2005; Phillpis,1991, 2006; Besette et el., 2007; Garnweidner, 2010; Beug et al., 2014; Kuo and Methven, 2014). The determined macrofungi samples are kept in the fungarium of Biology Department, Scince Faculty, Van Yüzüncü Yıl Üniversity. 


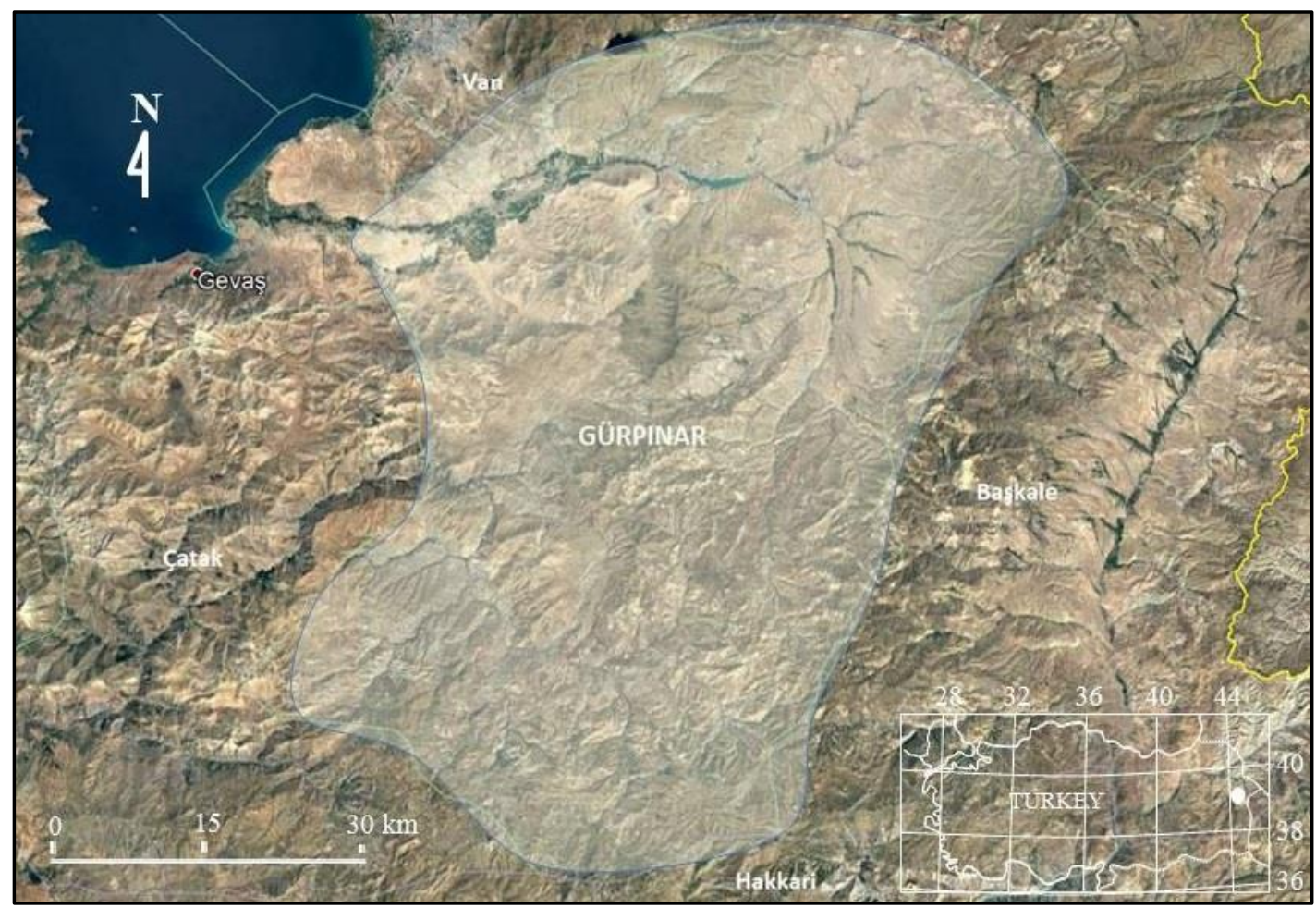

Figure 1. Map of the research area (modified from google earth)

\section{Results}

The list of the determined edible taxa, are given together with their habitats, collection localities, collection dates and the personal voucher numbers. The systematics of the taxa are in accordance with Kirk et al. (2008) and Index fungorum (accessed on 10 December 2018).

\section{Ascomycota Whittaker \\ Pezizomycetes O.E. Erikss. \& Winka \\ Pezizales J. Schröt. \\ Helvellaceae Fr.}

1. Helvella acetabulum (L.) Quél.: Among needle litter under Pinus L. sp. trees, locality 14, 18.5.2015, Şelem 71.

2. Helvella lacunosa Afzel: Among leaf litter under Populus sp. trees, locality 14, 5.6.2016, Şelem 132.

3. Helvella leucopus Pers.: Among leaf litter under Populus sp. trees, locality 14, 18.5.2015, Şelem 116.

4. Paxina queletii (Bres.) Stangl: Under Salix sp. trees, locality 14, 5.10.2016, Şelem 131.

\section{Morchellaceae Rchb.}

5. Mitrophora semilibera (DC.) Lév.: Among leaf litter under Populus sp. trees, locality 14, 18.05.2015, Şelem 63; under Salix sp. trees, locality 6, 05.10.2016, Şelem 172.

6. Morchella elata Fr.: Among leaf litter under Populus sp. trees, locality 11, 03.06.2016, Şelem 230 .

7. Morchella esculenta (L.): Pers.: Among leaf litter under Populus sp. trees, locality 11, 03.06.2016, Şelem 83; under Salix sp. trees, locality 9, 08.05.2015, Şelem 11.

8. Morchella esculentoides M. Kuo, Dewsbury, Moncalvo \& S.L.Stephenson: Among leaf litter under Populus and
Salix sp. trees, locality 11, 03.06.2016, Şelem 96.

9. Morchella prava Dewsbury, Moncalvo, J.D. Moore \& M.Kuo: Among leaf litter under Populus and Salix sp. trees, locality 11, 03.06.2016, Şelem 94.

Basidiomycota Whittaker ex Moore

Agaricales Underw.

Agaricaceae Chevall.

10. Agaricus bisporus (J.E. Lange) Imbach : Among grass in meadow, locality 9, 18.05.2015, Şelem 98.

11. Agaricus campestris L.: Among grass in meadow, locality 13, 18.05.2015, Şelem 21.

12. Agaricus urinascens (Jul. Schäff. \& F.H.Møller) Singer: Among grass in meadow, locality 12, 5.10.2016, Şelem 316.

13. Bovista plumbea Pers.: Among grass in meadow, locality 10, 30.05.2017, Şelem 360 .

14. Coprinus comatus (O.F. Müll.) Pers.: Among grass in meadow, locality 30, 18.05.2015, Şelem 51; locality 19, 03.06.2016, Şelem 248; under Salix sp. trees, locality 24, 03.06.2016, Şelem 225.

\section{Hymenogastraceae Vittad.}

15. Psilocybe coronilla (Bull.) Noordel. : Among grass in meadow, locality 9, 18.05.2015, Şelem 92; locality 36, 18.11.2015, Şelem 196.

\section{Pleurotaceae Kühner}

16. Pleurotus eryngii (DC.) Quél.: On Ferula sp. remains, locality 33, 03.06.2016, Şelem 242; locality 18, 28.06.2017, Şelem 354; locality 1, 13.06.2017, Şelem 365; locality 2, 13.06.2017, Şelem 375; locality 7, 13.06.2017, 
Table 1. Macrofungi collection localities

\begin{tabular}{|c|c|c|c|}
\hline $\begin{array}{c}\text { Locality } \\
\text { No }\end{array}$ & Locality name & Coordinates & Altitude \\
\hline 1 & Albenek village & $\mathrm{N} 38^{\circ} 16.015^{\prime} ; \mathrm{E} 43^{\circ} 37.050^{\prime}$ & $2022 \mathrm{~m}$ \\
\hline 2 & Alnıak village & N $38^{\circ} 13.126^{\prime} ;$ E $43^{\circ} 42.047^{\prime}$ & $2502 \mathrm{~m}$ \\
\hline 3 & Bozyiğit village & N $38^{\circ} 21.965^{\prime} ;$ E $43^{\circ} 34.098^{\prime}$ & $1845 \mathrm{~m}$ \\
\hline 4 & Çörekli village & N $38^{\circ} 21.147^{\prime} ;$ E $43^{\circ} 48.146^{\prime}$ & $2001 \mathrm{~m}$ \\
\hline 5 & Between Çörekli- Cevizalan villages & $\mathrm{N} 38^{\circ} 21.855^{\prime} ; \mathrm{E} 43^{\circ} 48.062^{\prime}$ & $2025 \mathrm{~m}$ \\
\hline 6 & Erkald 1 village & $\mathrm{N} 38^{\circ} 21.842^{\prime} ; \mathrm{E} 43^{\circ} 33.202^{\prime}$ & $1809 \mathrm{~m}$ \\
\hline 7 & Giyimli village & $\mathrm{N} 38^{\circ} 12.040^{\prime} ; \mathrm{E} 43^{\circ} 47.090^{\prime}$ & $2322 \mathrm{~m}$ \\
\hline 8 & Günbaş1 village & $\mathrm{N} 38^{\circ} 18.300^{\prime} ; \mathrm{E} 43^{\circ} 43.051^{\prime}$ & $2168 \mathrm{~m}$ \\
\hline 9 & Gürpınar central county & $\mathrm{N} 38^{\circ} 19.820^{\prime} ; \mathrm{E} 43^{\circ} 25.010^{\prime}$ & $1745 \mathrm{~m}$ \\
\hline 10 & Gürpınar central county & N $38^{\circ} 19.534^{\prime} ;$ E $43^{\circ} 23.867^{\prime}$ & $1751 \mathrm{~m}$ \\
\hline 11 & Around Gürpınar & N $38^{\circ} 17.929^{\prime} ;$ E $43^{\circ} 45.243^{\prime}$ & $2010 \mathrm{~m}$ \\
\hline 12 & Güzelsu village & N $38^{\circ} 18.960^{\prime} ;$ E $43^{\circ} 48.130^{\prime}$ & $1980 \mathrm{~m}$ \\
\hline 13 & Güzelsu village & N $38^{\circ} 17.925^{\prime} ;$ E $43^{\circ} 48.828^{\prime}$ & $1996 \mathrm{~m}$ \\
\hline 14 & Güzelsu village & N $38^{\circ} 19.303^{\prime} ;$ E $43^{\circ} 47.973^{\prime}$ & $1977 \mathrm{~m}$ \\
\hline 15 & Güzelsu village, near castle & N $38^{\circ} 19.139^{\prime} ;$ E $43^{\circ} 47.991^{\prime}$ & $1986 \mathrm{~m}$ \\
\hline 16 & Hacıköy village & $\mathrm{N} 38^{\circ} 18.093^{\prime} ; \mathrm{E} 43^{\circ} 38.100^{\prime}$ & $2205 \mathrm{~m}$ \\
\hline 17 & Hamurkesen village & N $38^{\circ} 20.150^{\prime} ;$ E $43^{\circ} 37.650^{\prime}$ & $1948 \mathrm{~m}$ \\
\hline 18 & Işıkpınar village & $\mathrm{N} 38^{\circ} 19.450^{\prime} ; \mathrm{E} 43^{\circ} 36.840^{\prime}$ & $2134 \mathrm{~m}$ \\
\hline 19 & Kırgeçit village & N $38^{\circ} 11.320^{\prime} ;$ E $43^{\circ} 29.884^{\prime}$ & $2147 \mathrm{~m}$ \\
\hline 20 & Kırgeçit village & N $38^{\circ} 11.321^{\prime} ;$ E $43^{\circ} 29.884^{\prime}$ & $2165 \mathrm{~m}$ \\
\hline 21 & Kuşdağı place & $\mathrm{N} 38^{\circ} 14.690^{\prime} ; \mathrm{E} 43^{\circ} 27.380^{\prime}$ & $1905 \mathrm{~m}$ \\
\hline 22 & Ortaköy village & $\mathrm{N} 38^{\circ} 21.873^{\prime} ; \mathrm{E} 43^{\circ} 38.294^{\prime}$ & $1910 \mathrm{~m}$ \\
\hline 23 & Ortaköy village & N $38^{\circ} 21.734^{\prime} ;$ E $43^{\circ} 37.854^{\prime}$ & $1868 \mathrm{~m}$ \\
\hline 24 & Örmeli village & $\mathrm{N} 38^{\circ} 07.220^{\prime} ; \mathrm{E} 43^{\circ} 30.430^{\prime}$ & $2231 \mathrm{~m}$ \\
\hline 25 & Sevindik village & N $38^{\circ} 18.293^{\prime} ;$ E $43^{\circ} 52.059^{\prime}$ & $2098 \mathrm{~m}$ \\
\hline 26 & Sevindik village & N $38^{\circ} 17.972^{\prime} ;$ E $43^{\circ} 50.348^{\prime}$ & $2038 \mathrm{~m}$ \\
\hline 27 & Taşdöndüren village & N $38^{\circ} 15.328^{\prime} ;$ E $43^{\circ} 48.910^{\prime}$ & $2031 \mathrm{~m}$ \\
\hline 28 & Tepegören village & N $38^{\circ} 21.506^{\prime} ;$ E $43^{\circ} 53.276^{\prime}$ & $2107 \mathrm{~m}$ \\
\hline 29 & Tutmaç village & N $38^{\circ} 15.930^{\prime} ;$ E $43^{\circ} 42.790^{\prime}$ & $2401 \mathrm{~m}$ \\
\hline 30 & Üçgen village & N $38^{\circ} 22.515^{\prime} ;$ E $43^{\circ} 44.693^{\prime}$ & $2141 \mathrm{~m}$ \\
\hline 31 & Üçgen village & N $38^{\circ} 22.363^{\prime} ;$ E $43^{\circ} 44.975^{\prime}$ & $2135 \mathrm{~m}$ \\
\hline 32 & Yedisalkım village & N $38^{\circ} 11.012^{\prime} ;$ E $43^{\circ} 42.100^{\prime}$ & $2441 \mathrm{~m}$ \\
\hline 33 & Yoldüştü village & N $38^{\circ} 09.710^{\prime} ;$ E $43^{\circ} 33.790^{\prime}$ & $2187 \mathrm{~m}$ \\
\hline 34 & Yurtbaşı village & N $38^{\circ} 13.933^{\prime} ;$ E $43^{\circ} 47.250^{\prime}$ & $2108 \mathrm{~m}$ \\
\hline 35 & Around Zernek watchouse & N $38^{\circ} 21.562^{\prime} ;$ E $43^{\circ} 39.162^{\prime}$ & $1934 \mathrm{~m}$ \\
\hline 36 & Around Zernek watchouse & N $38^{\circ} 21.676^{\prime} ;$ E $43^{\circ} 38.620^{\prime}$ & $1900 \mathrm{~m}$ \\
\hline
\end{tabular}

Şelem 372; locality 29, 13.06.2017, Şelem 377; locality 32, 13.06.2017, Şelem 374.

17. Pleurotus ostreatus (Jacq.) P. Kumm.: On Populus sp. stump, locality 14, 18.05.2015, Şelem 54; locality 14, 05.06.2015, Şelem.120; locality 10, 08.11.2015, Şelem 180 ; locality $10,18.11 .2015$, Şelem 211 ; locality 4, 13.11.2016, Şelem 382; locality 26, 13.11.2016, Şelem 384; on Salix sp. stump locality 27, 13.06.2017, Șelem 368; locality 28, 13.06.2017, Şelem 367; locality 34, 13.06.2017, Şelem 371.

18. Pleurotus populinus O.Hilber \& O.K.Mill.: On Populus sp. stump, locality 15, 05.10.2016, Şelem 317.

Pluteaceae Kotl. \& Pouzar

19. Pluteus aurantiorugosus (Trog) Sacc.: On Populus sp., stump, locality 6, 19.05.2016, Şelem 338 .
20. Pluteus romellii (Britzelm.) Sacc.: On Salix sp. stump, locality 14, 18.05.2015, Şelem 55; on Populus sp. stump, locality 12, 05.10.2016, Şelem. 300.

21. Volvopluteus gloiocephalus (DC.) Vizzini, Contu \& Justo: Among grass in meadow, locality 9, 18.05.2015, Şelem 109.

Psathyrellaceae Vilgalys, Moncalvo \& Redhead

22. Coprinellus disseminatus (Pers.) J.E. Lange: Around Populus and Salix sp. trees, locality 15, 18.05.2015, Şelem 28; locality 22, 18.05.2015, Şelem 76; locality 23, 18.11.2015, Şelem 185.

23. Coprinellus micaceus (Bull.) Vilgalys, Hopple \& Jacq. Johnson: Under Salix sp. trees, locality 9, 08.05.2015, Şelem 3; under Populus sp. trees, locality 12, 18.05.2015, Şelem 29; locality 30, 03.06.2016, Şelem 225; 
locality 31, 03.06.2016, Şelem 227; locality 21, 03.06.2016, Şelem 252; locality 17, 28.06.2017, Şelem 351; locality 8, 28.06.2017, Şelem 353 .

24. Coprinopsis atramentaria (Bull.) Redhead, Vilgalys \& Moncalvo: Around Populus sp. stump, locality 14, 05.06.2015, Şelem 126; locality 23, 18.11.2015, Şelem 183; locality 5, 03.06.2016, Şelem 364; around Salix sp. stump, locality 15, 18.05.2015, Şelem 39; under Robinia sp. tree, locality 22, 218.05.2015, Şelem 88 .

25. Psathyrella candolleana (Fr.) Maire: Around Populus and Salix sp. trees, locality 14, 05.06.2015, Şelem 123; locality 14, 05.06.2015, Şelem 173 .

\section{Strophariaceae Singer \& A.H.Sm.}

26. Agrocybe dura (Bolton) Singer: Among grass in meadow, locality 16, 28.06.2017. Şelem 361.

27. Agrocybe pediades (Fr.) Fayod: Among grass in meadow, locality 36, 18.05.2015, Şelem 72 .

28. Agrocybe praecox (Pers.) Fayod: Among grass in meadow, locality 14, 05.06.2015, Şelem 58.

29. Cyclocybe cylindracea (DC.) Vizzini \& Angelini: On Populus sp. stump, locality 11, 05.10.2016, Şelem 268.

30. Pholiota aurivella (Batsch) P. Kumm.: On Salix sp. stump, locality 3, 18.11.2015, Şelem 210; locality 10, 18.11.2015, Şelem 214; locality $14,05.10 .2016$, Şelem 288; locality 25, 13.11.2016, Şelem 383.

\section{Tricholomataceae Lotsy}

31. Melanoleuca brevipes (Bull.) Pat.: Among grass in meadow, locality 14, 18.05.2015, Şelem 52; among grass under Populus sp. trees, locality 10], 19.05.2016, Şelem 326.

32. Melanoleuca cognata (Fr.) Konrad \& Maubl.: Among grass under Salix sp. tree, locality 12, 05.10.2016, Şelem 309.

33. Pseudoclitocybe cyathiformis (Bull.) Singer: Among grass in meadow, locality 22, 18.11.2015, Şelem 193.

34. Lepista personata (Fr.) Cooke: Among grass in meadow, locality 20, 03.06.2016, Şelem 247.

Boletales E.-J.Gilbert

Suillaceae Besl \& Bresinsky

35. Suillus collinitus (Fr.) Kuntze : Among needle litter under Pinus sp. trees, locality 35, 18.11.2015, Şelem 192.

\section{Polyporales Gäum}

Polyporaceae Fr. ex Corda

36. Cerioporus squamosus (Huds.) Quél.: On Populus sp. stump, locality 15, 18.05.2015, Şelem 356.

\section{Discussions}

As a result of this study, 36 wild edible macrofungi taxa belonging to 11 families, 4 orders and 2 classes were determined within the boundaries of Gürpınar district. Eight of the determined species belong to Ascomycota and 28 belong to Basidiomycota. Though 36 edible macrofungi exist in the region, it is found that only six of them are collected and consumed in the region (Table 2). This benefit rate (\%16.6) seems to be smaller than the average benefit rate of naturally growing wild edible mushrooms in Turkey (Table 3).

Table 2. Locally consumed taxa and their local names

\begin{tabular}{ll}
\hline Scientific name & Local name \\
\hline Agaricus bisporus & Çayır mantarı \\
Agaricus campestris & Çayır mantarı \\
Agaricus urinascens & Çayır mantarı \\
Pleurotus eryngii & Heliz mantarı \\
Pleurotus ostreatus & Kavak mantarı \\
Pleurotus populinus & Kavak mantarı \\
\hline
\end{tabular}

Among the determined edible species, Pleurotus eryngii is the most preferable one due to its delicious taste and the belief that it is good for indigestion. It is also important for its economic potential. Especially in May and June this fungus is collected and sold in public bazaars and markets. It had a price of 50 Turkish liras per kilogram in 2018.

Collection and consumption of the members of the genus Agaricus L. also common in the region with the name meadow mushroom (çayır mantarı). Coprinopsis atramentaria was also determined in the region. Normally this mushroom is an edible one, but if it is consumed with alcohol, it becomes poisonous due to the interaction of coprine with alcohol (Michelot, 1992).

Though regarded as edible, Helvella acetabulum, $H$. lacunosa, Morchella prava and Pholiota aurivella should not be consumed unless cooked. Among them $H$. acetabulum, $H$. lacunosa, Paxina queletii and $P$. aurivella may cause gastrointestinal distress or gastric upset following ingestion (Gücin et al., 2000). Since it liquifies at maturity, Coprinus comatus should be consumed when young and still white. Likewise, Pholiota aurivella and Cerioporus squamosus should also be consumed when young, before the fruit bodies become hard and ligneous.

Pluteus aurantiorugosus is an attractive mushroom and could be regarded as suspecious due to its red to yellowish colour, but it is used for food in Mexica and Malaysia (Boa, 2004).

Wild edible mushrooms are among the most valuable nonwood forest products and have been collected and consumed by people for thousands of years (Boa, 2004). But, it should be avoided to eat naturally growing mushrooms if the edibility is not definitely known.

\section{Acknowledgments}

Authors would like to thank Yüzüncü Y1l University Research Projects Presidency (FYL-2016-5213) for its financial support. 
Table 3. Local consumption percentages of wild edible mushrooms in some regions of Turkey (modified from Kaya, 2018)

\begin{tabular}{lccr}
\hline \multirow{2}{*}{ Reseachs } & Number of edible taxa & Local consumption \\
\cline { 3 - 4 } & 27 & 5 & taxa number \\
\hline Demirel (1996) & 22 & 8 & 18,52 \\
Yildız and Ertekin (1997) & 49 & 26 & 36,36 \\
Solak et al. (1999) & 48 & 13 & 53,06 \\
Gezer (2000) & 21 & 2 & 27,08 \\
Demirel et al. (2002) & 60 & 6 & 9,52 \\
Demirel et al. (2003) & 47 & 7 & 10 \\
Demirel et al. (2015) & 25 & 5 & 14.89 \\
Kaya et al. (2012) & 32 & 7 & 23,09 \\
Atila and Kaya (2013) & 59 & 6 & 17,71 \\
Keleş et al. (2014) & 60 & 9 & 10,17 \\
Akata et al. (2014) & 54 & 7 & 15 \\
Uzun et al. (2015) & 40 & 6 & 12,97 \\
Kaya (2015) & 46 & 4 & 12 \\
Akata et al. (2016) & 45 & 15 & 8,7 \\
Uzun et al. (2017) & & & Average \\
\hline
\end{tabular}

\section{References}

Acharya K, Ghosh S, Biswas R (2018). Total phenolic contents and antioxidant effects of infusion and decoction from Lepista sordida (Schumach.) Singer. Fabad Journal of Pharmaceutical Sciences 43(1): 17-24.

Akata I, Uzun Y, Kaya A (2014). Macromycetes determined in Yomra (Trabzon) district. Turkish Journal of Botany 38: 999. 1012.

Akata I, Uzun Y, Kaya A (2016). Macrofungial diversity of Zigana Mountain. Biological Diversity and Conservation 9(2): 57-69. Anonymus (2018). Gürpınar Kaymakamlığı. http://www.gurpinar.gov.tr/ilcemiz-taniyalim/ [accessed 10 December 2018]. Atalay İ (1994). Türkiye Coğrafyası. İzmir: Ege Üniversitesi Basımevi.

Atila OY, Kaya A (2013). Macromycetes of Sarız (Kayseri - Turkey) district. Biological Diversity and Conservation 6(2): 50-54.

Bani B, Adıgüzel N (2008). Flora of an important plant area: Çatak valley I - (Çatak-Pervari) Van, Turkey. Flora Mediterranea 18: 11-63.

Besette AE, Roody WC, Besette AR, Dunaway DL (2007). Muhrooms of the Southeastern United States (first edition). SyracuseNew York: Syracuse University Press.

Bessette AE, Bessette AR, Fischer DW (1997). Mushrooms of Northeastren Nort America, Hong Kong: Syracuse University Press.

Beug M, Bessette AE, Bessette AR (2014). Ascomycete Fungi of North America. A Mushroom Reference Guide. Texas-USA: University of Texas Press.

Boa E (2004). Wild edible fungi: a global overview of their use and importance to people. NonWood Forest Products, No. 17, Rome: FAO, Forestry Department.

Breitenbach J, Kränzlin F (1984). Fungi of Switzerland, Vol. 1. Lucerne: Verlag Mykologia.

Breitenbach J, Kränzlin F (1991). Fungi of Switzerland, Vol. 3. Lucerne: Verlag Mykologia.

Breitenbach J, Kränzlin F (1995). Fungi of Switzerland, Vol. 4. Lucerne: Verlag Mykologia.

Breitenbach J, Kränzlin F (2000). Fungi of Switzerland, Vol. 5. Lucerne: Verlag Mykologia.

Buczacki S (1989). Fungi of Britain and Europe. Glasgow: William Collins and Sons Ltd.

Cappelli A (1984). Fungi Europaei 1. Agaricus. Saronno-Italia: Liberia editrice Biella Giovanna..

Demirbaş A (2001). Concentrations of 21 Metals in 18 species of mushrooms growing in the East Black Sea Region. Food Chemistry 75: 453-457.

Demirel K (1996). Van Yöresi Makrofungusları. Tr. J. of Botany, 20: 165-169.

Demirel K, Kaya A, Uzun Y (2003). Macrofungi of Erzurum Province. Turkish Journal of Botany 27: 29-36.

Demirel K, Uzun Y, Akçay ME, Keleş A, Acar İ, Efe V (2015). Van Yöresi Makromantarlarına Katkılar. Mantar Dergisi 6(2): 1223.

Demirel K, Uzun Y, Kaya A (2002). Macrofungi of Ağrı Province. Turkish Journal of Botany 26: 291-295.

Dündar Ö, Demircioğlu H, Özkaya O, Dündar B (2016). Kültür mantarlarının muhafazası ve kalite özellikleri üzerine yapılan araştırmalar. Turkish Journal of Agriculture: Food Science And Technology 4(3): 150-154.

Garnweidner E (2010). Mushrooms and Toadstools of Britain and Europe. London: Harper Collins. 
Gezer K (2000). Contributions to the Macrofungi Flora of Antalya Province. Turkish Journal of Botany 24: 293-298.

Gücin F, Işıloğlu M, Kaya A (2000). Türkiye'de Zehirli Mantarlar. In: Mat A (ed.) Türkiye'de Mantar Zehirlenmeleri Zehirli Mantarlar. İstanbul: Nobel Tip Kitabevleri Ltd., pp 9-150.

Index Fungorum (2018). http://www.indexfungorum.org/names/Names.asp / [10 December 2018].

Işı1ldak Ö, Turkekul İ, Elmastaş M, Tuzen M (2004). Analysis of heavy metals in some wild-grown edible mushrooms from the middle black sea region, Turkey. Food Chemistry 86(4): 547-552.

Jordan M (1995). The Encyclopedia of Fungi of Great Britain and Europe. UK: David and Charles Book Co.

Kalač P (2010). Trace element contents in European species of wild growing edible mushrooms: A review for the period $2000-$ 2009. Food Chemistry 122(1): 2-15.

Karapınar HS, Uzun Y, Kılıçel F (2017). Mineral contents of two wild morels. Anatolian Journal of Botany 1(2): 32-36.

Kaya A (2015). Contributions to the Macrofungal Diversity of Atatürk Dam Lake Basin. Turkish Journal of Botany 39: 162-172.

Kaya A (2018). Edible Macrofungi Determined in Adıyaman Province (Turkey). International Eurasian Conference on Science, Engineering and Technology (EurasianSciEnTech 2018), November 22-23, 2018 Ankara, Turkey. Proceeding book pp. 461-467.

Kaya A, Bağ H (2013). Mineral contents of some wild ascomycetous mushrooms. Asian Journal of Chemistry 25: 1723-1726.

Kaya A, Demirel K, Uzun Y (2012). Macrofungal diversity of Araban (Gaziantep/Turkey) district. Biological Diversity and Conservation 5(3): 162-166.

Kaya A, Uzun Y (2018). New contributions to the turkish ascomycota. Turkish Journal of Botany 42: 644-652.

Keleş A, Demirel K, Uzun Y, Kaya A (2014). Macrofungi of Ayder (Rize/Turkey) high plateau. Biological Diversity and Conservation 7(3): 177-183.

Kirk PM, Cannon PF, Minter DW, Stalpers JA (2008). Dictionary of the Fungi. 10th ed. Wallingford, UK: CAB International.

Korkmaz ME, Ağar O, Kaya A, Emsen B, Uzun Y (2016). Measurement of gross alpha and beta activities in mushroom samples. Cumhuriyet University Faculty of Science Science Journal 37(4): 325-328.

Kränzlin F (2005). Fungi of Switzerland, Vol. 6. Lucerne: Verlag Mykologia.

Kuo M, Methven SA (2014). Mushrooms of the Midwest. Chicago: University of Illinois Press.

Manzi P, Aguzzi A, Pizzoferrato L (2001). Nutritional Value of Mushrooms Widely Consumed in Italy. Food Chemistry 73: 321325.

Mat A (2000). Türkiye'de Mantar Zehirlenmeleri ve Zehirli Mantarlar. İstanbul: Nobel Tıp Kitabevleri Ltd.

Michelot D (1992). Poisoning by Coprinus atramentarius. Natural Toxins 1(2): 73-80.

Moser M (1983). Keys to Agarics and Boleti. Stuttart: Gustav Fiscer Verlag.

Owaid MN, Muslat MM, Abed IA (2017). Farklı kompostlarda üretilen Agarıcus bisporus X25 mantarlarının protein ve ortodihidrik fenol içerikleri. Gıda 42(3): 324-328.

Phillips R (2006). Mushrooms. London: Pan Macmillan.

Phillips R, Foy N, Kibby G (1991). Mushrooms of North America. USA: Little Brown and Co.

Solak MH, Işıloğlu M, Gücin F, Gökler İ (1999). Macrofungi of İzmir Province. Turkish Journal of Botany 23: 383-390.

Soylak M, Saraçoğlu S, Tüzen M, Mendil D (2005). Determination of trace metals in mushroom samples from Kayseri, Turkey. Food Chemistry 92(4): 649-652.

Türkekul İ (2017). Yenebilen ve ekonomik değeri olan Ramaria flavobrunnescens= Gelinparmağı Mantarı'nın vitamin C, E ve yağ asidi bileşenlerinin belirlenmesi. Kahramanmaraş Sütçü İmam Üniversitesi Doğa Bilimleri Dergisi 20(1): 16-19.

Türkmen M, Budur D (2018). Heavy metal contaminations in edible wild mushroom species from Turkey's Black Sea region. Food chemistry 254: 256-259.

Uzun Y, Acar İ, Akçay ME, Kaya A (2017). Contributions to the macrofungi of Bingöl, Turkey. Turkish Journal of Botany 41: 516-534.

Uzun Y, Gençcelep H, Kaya A, Akçay ME (2011). The mineral contents of some wild edible mushrooms. Ekoloji 80: 6-12.

Uzun Y, Kaya A, Karacan İH, Kaya ÖF, Yakar S (2015). Macromycetes determined in Islahiye (Gaziantep/Turkey) district. Biological Diversity and Conservation 8(3): 209-217.

Yildız A, Ertekin AS (1997). Contributions to The Macrofungal Flora of Diyarbakır, Turkish Journal of Botany 21: 119-122.

Cite this article: Şelem E, Keleş A, Acar İ, Demirel K (2019). Edible macrofungi determined in Gürpınar (Van) district. Anatolian Journal of Botany 3(1): 7-12. 